\title{
Erratum: Changes in Shape of the Combination of a One-Length Cut and a Medium-Graduation Cut according to Segmentation of Head Regions and Slice Lines
}

Seon-Mi Jang, Won-Ji Jung*

Department of Beauty Science, Kwangju Women's University, Kwangju, Korea

Erratum to: Asian J Beauty Cosmetol 2021; 18(2): 221-233 (http://dx.doi.org/10.20402/ajbc.2020.0027)

The article 'Changes in Shape of the Combination of a One-Length Cut and a Medium-Graduation Cut according to Segmentation of Head Regions and Slice Lines' authored by Seon-Mi Jang, Won-Ji Jung, published in June issue [Vol 18, No 2] of Asian Journal of Beauty and Cosmetology (2020), has an erratum.

On Table 6 of page 227, there was a misspelling. The 'A line' should be written as 'V line'.

The Asian Journal of Beauty and Cosmetology apologizes to the readers for this error. 